\title{
Measurement of edge plasma parameters at W7-X using Alkali Beam Emission Spectroscopy
}

\author{
G. Anda ${ }^{1}$, D. Dunai ${ }^{1}$, T. Krizsanóczi ${ }^{1}$, D. Nagy ${ }^{1}$, M. Otte ${ }^{2}$, S. Hegedüs ${ }^{1}$, M. Vécsei ${ }^{1}$, S. Zoletnik $^{1}$, \\ G. Gárdonyi ${ }^{2}$ and the $\mathrm{W} 7-\mathrm{X}$ team $^{3}$ \\ ${ }^{1}$ Wigner RCP, Association EURATOM, Budapest, Hungary \\ ${ }^{2}$ Budapest University of Technology and Economics \\ Budapest, Hungary \\ ${ }^{3}$ IPP Greifswald, Germany
}

\begin{abstract}
A $60 \mathrm{keV}$ neutral Alkali beam system was designed, built and installed for beam emission spectroscopy measurement of edge plasma on W7-X.

As the decay length of the first excited state of sodium is much shorter than lithium it was selected as beam species. This way the steep density gradient at the bean shaped cross section of the Wendelstein 7-X plasma can be resolved.

Unexpectedly a thermal sodium beam was also observed in the far Scrape Off Layer of W7-X plasma during the first measurements in 2017. It was found to originate from the recirculating neutralizer which uses sodium vapour. To resolve this issue the neutralizer material was changed to Potassium.

The observation system consists of two parts: a 40 channel avalanche photo diode (APD) camera unit and a CMOS camera which run in parallel: $95 \%$ of the collected light goes to the APD unit which is digitized with $2 \mathrm{MHz}$ sampling rate while the CCD camera is operated in the $100 \mathrm{~Hz}$ range. Chopping the beam up to $250 \mathrm{kHz}$ is also possible for precise background measurement on the time scale of the turbulence.

In this paper the main improvements of the sodium beam system using potassium for ion beam neutralization and the first measurement results are described.
\end{abstract}

Keywords: alkali beam emission spectroscopy, ion source

\section{Introduction}

Spatially and temporally well resolved electron density measurements at the plasma edge are of great importance in the study of magnetic fusion devices. There are only a limited number of non-perturbative diagnostic techniques for this task, most notably thermal He beam and reflectometry. The alkali Beam Emission Spectroscopy (BES) diagnostic has excellent time $(\mu \mathrm{s})$ and very good spatial $(<0.5 \mathrm{~cm})$ resolution at the same time.

Capabilities of accelerated lithium beam based BES diagnostics were demonstrated at almost every present tokamak and stellarator experiments (ASDEX [1], ASDEX Upgrade [2], JET [3], TEXTOR [4], DIII-D [5], W7-AS [6], LHD [7] and COMPASS-D [8]) and was designed and installed also at W7-X in year 2017. The working principle of the alkali beam based BES can be summarized as follows: alkali ions (lithium or sodium) are extracted from a thermionic ion source and accelerated by an ion optic. Neutralization of the ion beam is done in sodium vapour. The neutral beam penetrates the plasma and is excited by plasma particles. The excited state decays with photon emission at a characteristic wavelength. As the excitation is mainly determined by the plasma electron density, the alkali BES diagnostic measures the plasma electron density and its fluctuations [9]. For accurate background light correction the beam can be "chopped" from the plasma at a rate of $250 \mathrm{kHz}$.

Limitations of the diagnostic are the restricted penetration depth $(10-20 \mathrm{~cm})$ and the light intensity measured by the observation system. The latter depends on the neutral beam current and diameter, the optical observation system etendue and the efficiency of the detector system. Tuning of the interference filter by changing its temperature could also be very important.

Using Sodium alkali neutral beam a new limitation emerged. The sodium vapour used for ion beam neutralization appears as a thermal beam in the far Scrape Off Layer (SOL). As the sodium loss of the neutralizer can not be decreased a new material had to be chosen for ion beam neutralization (see in Section 2.3).

The article is organized as follows. Section 2.1 gives an overview of the injector hardware. In Section 2.2 the latest improvements of the system are summarized and in 2.3 the recirculating neutralizer is discussed. In Section 3 the test measurement with potassium filled neutralizer is shown. Section 4 shows the integration of the complete system at W7-X while in Section 4.1 and in 4.2 the observation system and the interference temperature tuner are described. In Section 5 the first measurements results are presented.

\section{Injector hardware}

\subsection{Overview of the system}

The alkali beam hardware is discussed in detail in Ref. [10] and only shortly summarized here. The arrangement of the W7-X alkali beam injector can be seen in Figure 1. The thermionic ion source is placed in a Pierce electrode and followed by a two-step ion optic, based on the original 
design used first at ASDEX [1]. The last element of the ion optic called puller is on $-500 \mathrm{~V}$ potential, i.e. it works as an electron suppression ring, as well. The ion beam enters into the deflection plates area where a pneumatic controlled miniature shutter is mounted between the electron suppression ring (puller) and the first deflection plate pair. The beam can be moved both poloidally and toroidally in this region by applying an electric field between the deflection plate pairs. A moveable Faraday cup is placed to measure the beam parameters.

The deflection area is followed by the recirculating neutralizer where the ion beam is neutralized - usually in sodium vapour. Due to lack of space, the diagnostic chamber could not be mounted at the entrance port, hence the system consists of two flight tubes (each of them with a length of about $0.5 \mathrm{~m}$, see in Fig. 1). At the diagnostic chamber a moveable Faraday cup and two observation cameras (with observation windows) are placed to measure the neutral beam parameters and the beam displacement from the beam axis, if there is any.

The injector is designed to produce a max. $60 \mathrm{kV}$ neutral alkali beam with about $1 \mathrm{~mA}$ neutral current and $2 \mathrm{~cm}$ FWHM. Additionally, the beam can either be chopped out completely (up to $250 \mathrm{kHz}$ ) or it is able to hop between two parallel beam positions in the plasma with about $400 \mathrm{kHz}$ frequency and a few $\mathrm{cm}$ from each other for poloidal flow velocity measurement [11].



Fig. 1. Overview of the W7-X alkali beam injector.

\subsection{Latest improvements of the beam hardware}

As mentioned before, one of the key parameters of the diagnostic is the measured light level, which highly depends on the beam focusing. The starting surface of the beam is critical, both its angle and its distance from the extractor. At earlier versions the main and the extractor voltage were insulated by two ceramic discs placed inside the vacuum chamber, as part of the holding structure of the Pierce electrode. In the present system the insulation of the main and extractor voltage is solved by a so called small ceramic break (see in Figure 1), while the main voltage and the ground is insulated by the main ceramic break. The holder structure of the Pierce electrode is stainless steel and is matched both on the flange and on the back side of the Pierce electrode, this way its position is fixed.

The high voltage system consists of two $60 \mathrm{kV}$ power supplies (Glassman, EJ60P10-F22-ETH) which are placed in a cabinet outside the torus hall. The HV system at the injector can be seen in Figure 2. There are load resistors $(100 \mathrm{MOhm}$ at the ion source, $45 \mathrm{MOhm}$ at the extractor), serial resistors $(90 \mathrm{kOhm}$ in both $\mathrm{HV}$ line to protect the system in case of HV sparks and discharges) and $\mathrm{HV}$ filter capacitors $(55 \mathrm{nF})$ to filter the ripple of the $\mathrm{HV}$ power supplies (only to each other). A new improvement is the HV transformer (made by Adimtech Ltd.). A toroidal iron core with primary windings $(n=520)$ is placed in a torus shaped UHMW polyethylene housing. The primary cables are let out from the HV area via a so-called chimney (that lies under the table shown in Fig. 2 ). The insulation does not break between the torus and the chimney. This way the secondary winding $(n=16$, not shown in Fig. 2) which is at the main voltage does not have to be insulated from the surrounding elements.



Fig. 2. HV system of W7-X alkali beam injector.

\subsection{Recirculating neutralizer}

Alkali ions extracted from the ion source and accelerated by the ion optic need to be neutralized in a sodium vapour cell via the charge exchange process. The concept of this kind of neutralizer is to heat up sodium, produce sodium vapour pressure in a cell, and minimize the loss of sodium by condensing and reflowing the sodium vapour (outside the neutralization volume) [10].

The loss of the neutralization can be calculated as follows:

$$
\varphi=n_{v} v \frac{r^{4} \pi}{2 L^{2}}
$$

where $n_{v}$ is the density and $v$ is the thermal velocity of Sodium atoms, $r$ is the diameter of the hole on the central cell and $L$ is the length of the neutralizer middle cell [10]. This equation results about $7.5 \mathrm{e}^{17} \mathrm{~s}^{-1}$ particle flux at both ends of the neutralizer. As a first attempt one can assume that the thermal beam leaves the neutralizer with an angle which is defined by the opening of the neutralizer $(25$ $\mathrm{mm})$, the opening of the middle cell $(25 \mathrm{~mm})$ and their distance $(0.1 \mathrm{~m})$. The distance between the neutralizer and the plasma edge is about $3 \mathrm{~m}$ and the entrance port 
diameter is about $63 \mathrm{~mm}$. It means that a thermal beam with about $1.3 \mathrm{e}^{16 \mathrm{~s}^{-1}}$ particle flux reaches the plasma.

As the measured extracted beam current was about 1.6-1.8 $\mathrm{mA}$ during OP1.2a and taking into account the about $40 \%$ focusing and about $80 \%$ neutralization efficiency the real neutral beam current could have been about $0.5 \mathrm{~mA}$. That would mean about a particle flux equal to $3 \mathrm{e}^{15} \mathrm{~s}^{-1}$. Such value is 4 times smaller than the thermal beam particle flux and this is not in agreement with the measured light level of the thermal beam, which is about one order of magnitude higher than the light of the accelerated beam. A possible explanation for this discrepancy is an under-estimation of the neutralizer temperature. It would in fact be enough to assume a $10 \%$ larger temperature to get a thermal particle flux of $3.7 \mathrm{e} 16$ $\mathrm{s}^{-1}[12]$.

The measured (by the CMOS camera) accelerated and thermal beam can be seen in Figure 3.

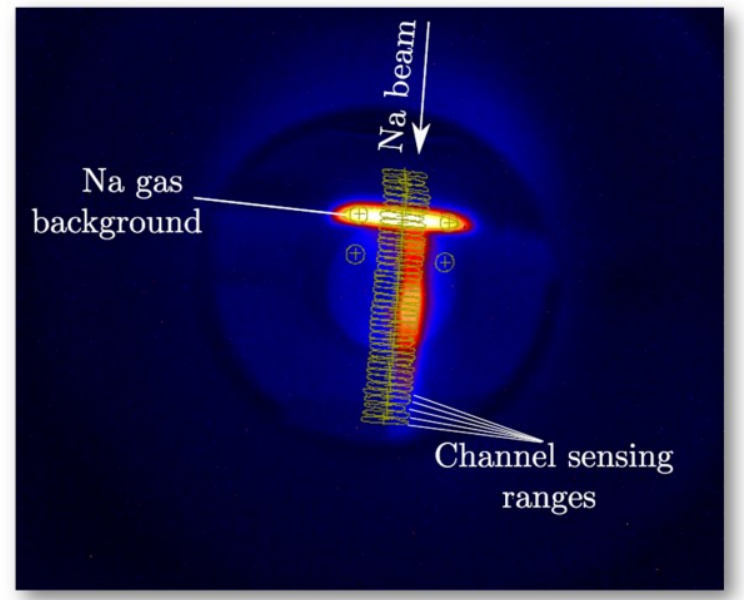

Fig. 3. CMOS camera measurement with the accelerated and thermal sodium beam.

Although additional investigations are necessary to describe accurately the light level measurement the consequence is clear: sodium vapour can not be used for neutralization of accelerated sodium beam as the light level originated from the thermal beam dominates the one due to the accelerated neutral beam. Instead of sodium two other alkali might be used, either lithium or potassium. Assuming the same cross section for the charge exchange interaction, to reach the same vapour pressure with lithium would require about $560^{\circ} \mathrm{C}$ degrees while with potassium that would take about $210^{\circ} \mathrm{C}$ degrees. This way potassium was chosen as an alternative for sodium beam neutralization.

\section{Neutralization test with potassium}

The clone of the W7-X alkali beam have been built up in Wigner RCP. Its neutralizer was filled up with about $6.7 \mathrm{~g}$ potassium (98\% purity). The beam diagnostic chamber was placed directly after the neutralizer chamber, without any flight tube.

Beam energy and $\mathrm{HV}$ ratio $\left(\mathrm{U}_{\text {main }} / \mathrm{U}_{\text {ext }}\right)$ were fixed at $40 \mathrm{kV}$ and at 9, where the sodium beam current had a maximum in the Faraday cup. The results can be seen in Figure 4.
At above $180^{\circ} \mathrm{C}$ degrees the neutralization efficiency reached almost $100 \%$ which shows very good agreement with the mentioned vapour pressure of potassium in Section 2.3.

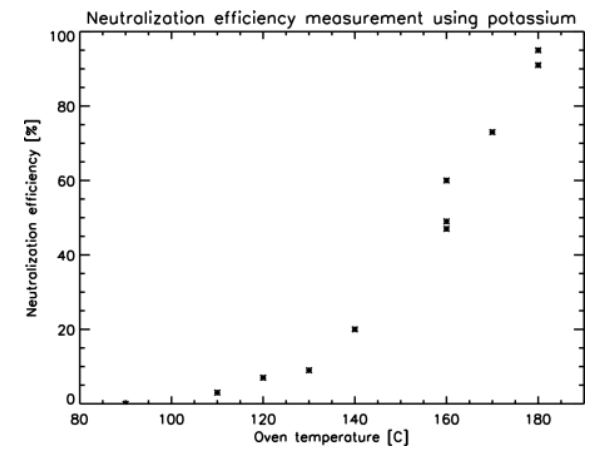

Fig. 4. Neutralization efficiency of a $40 \mathrm{keV}$ energy sodium beam using potassium vapour.

\section{Integration of the system}

The injector is placed between the two NBI systems at section 5. The entrance port of the neutral beam is AEA21 while the observation system located in the same cross section using port AEB20, see Figure 5.

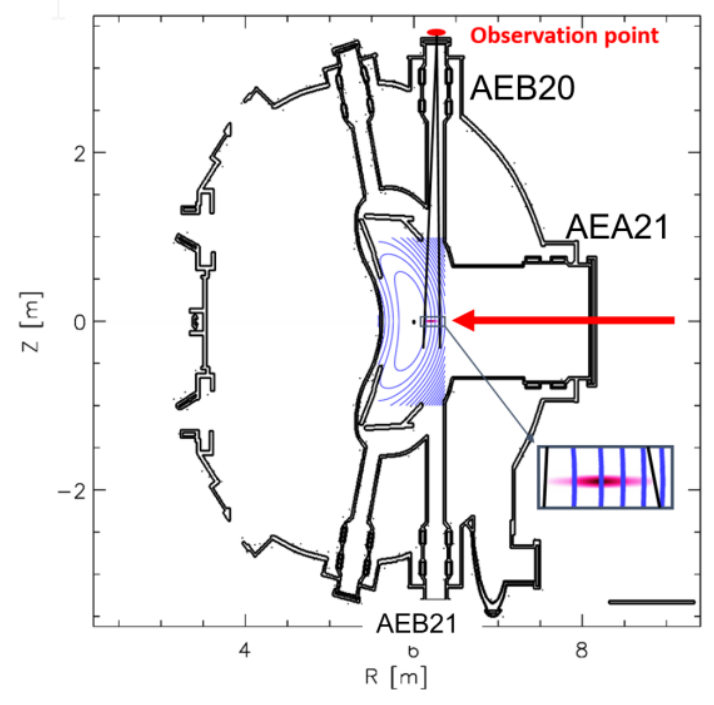

Fig. 5. Integration of the alkali BES at W7-X.

\subsection{Observation system}

The beam emission is observed from the poloidal direction with a high-etendue $\left(\Omega / 4 \pi=3 \times 10^{-4}\right) 40$ channel optical system, where each channel collects light from a $4 \times 0.5 \mathrm{~cm}^{2}$ (toroidal $\mathrm{x}$ radial) area of the beam to an avalanche photodiode system (APDCAM, Fusion Instruments Ltd.). To achieve sufficiently high photon flux the optics contains relatively big lenses (up to 244 $\mathrm{mm}$ diameter). The majority of the light is detected by the APDCAM system with $2 \mathrm{MHz}$ sampling rate while about $2.5 \%$ of the light is measured by a CMOS camera (PhotonFocus, 1280x1024) for overview and spatial calibration purposes. Despite the $500 \mathrm{kHz}$ analogue bandwidth the APD system has a peak signal-to-noise ratio up to 50, enabling the study of fast transients and turbulence. 


\subsection{Interference filter temperature control}

Special attention was paid to filter the beam light emission. The Sodium light emission occurs at two intensive spectral lines separated by $0.5 \mathrm{~nm}$ around 589 nm. A Carbon II line is located exactly on one of the Sodium lines, therefore cutting the Carbon emission was possible by measuring only one of the emission lines. To allow flexibility of the setup a filter was ordered which can be temperature tuned so as that room temperature it transmits both Sodium lines, while at about $60^{\circ} \mathrm{C}$ the Carbon line and one of the Sodium lines is strongly cut.

Designing a precise temperature control unit (to reach uniform temperature on the whole surface) for the 230 $\mathrm{mm}$ diameter filter was a special challenge. Its solution can be seen in Figure 6. Due to the big diameter the filter is composed by four pieces (by Andover Corp.) that are glued together onto an aluminum fixing frame plate. Heat is produced by 8 resistors ( $47 \mathrm{Ohm}, 10 \mathrm{~W}$ each) which are mounted on heat sinks. The heat is distributed by fans which circulate hot air between the filter and double windows (with antireflexion coating).

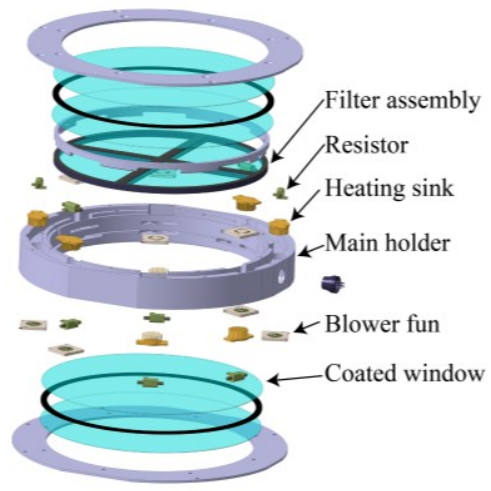

Fig. 6. Temperature control of the interference filter.

\section{First measurement results}

Although launch of the alkali BES system was planned for OP1.2b it was possible to start the measurements during OP1.2a, first with sodium vapour neutralization. For OP1.2b it was changed to potassium. An example CMOS camera image is shown in Figure 7. The thermal sodium beam background disappeared completely, as expected.

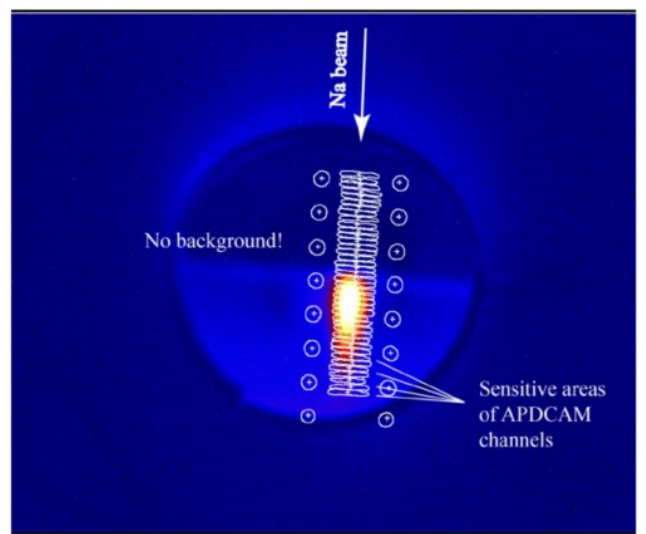

Fig. 7. CMOS camera measurement.
Results of the fast measurement system is shown in Figure 8. The electron density is reconstructed using the Bayesian method [13].

A density peak can be seen in the island region of W7-X. The reconstructed density profile agrees reasonably well with the magnetic surface structure [14], this way the diagnostic is capable of analyzing the density profiles and fluctuations in the magnetic island, as well.

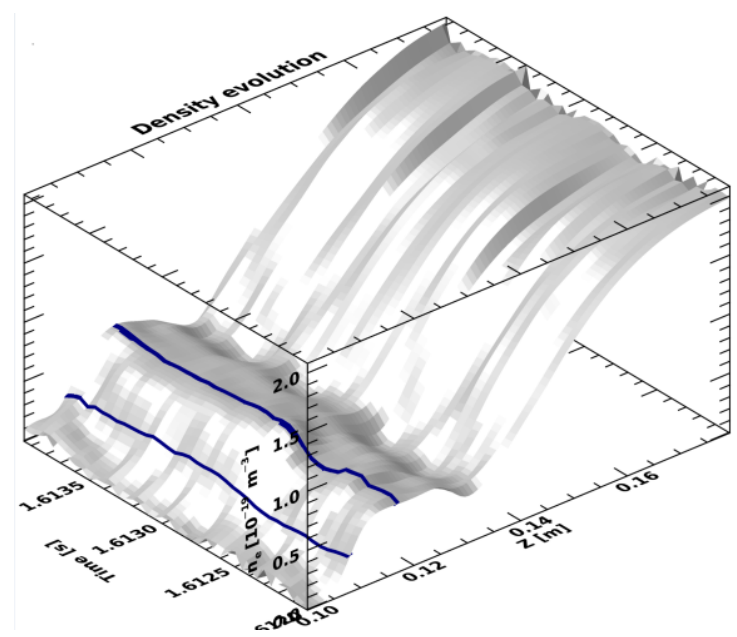

Fig. 8. Fast density reconstruction by the APDCAM detector.

\section{Acknowledgments}

This work has been carried out within the framework of the EUROfusion Consortium and has received funding from the Euratom research and training programme 2014-2018 under grant agreement No 633053. The views and opinions expressed herein do not necessarily reflect those of the European Commission.

\section{References}

[1] K. McCormick et al, Review of Scientific Instruments 56 (1985) 1063.

[2] M. Willensdorfer et al, Plasma Physics and Controlled Fusion 56 (2014) 025008.

[3] M. Brix at al, Review of Scientific Instruments 83 (2012) 10D533.

[4] E. Wolfrum et al, Review of Scientific Instruments 64 (1993) 2285.

[5] D. M. Thomas et al, Review of Scientific Instruments 63 (1992) 4940.

[6] S. Fiedler et al, Journal of Nuclear Materials 266-269 (1999) 1279.

[7] T. Morisaki et al, Review of Scientific Instruments 74 (2003) 1865.

[8] G. Anda et al, Fusion Engineering and Design 108 (2016) 16

[9] S. Zoletnik et al, Physics of Plasmas 6 (1999) 4239.

[10] G. Anda et al, Review of Scientific Instruments 89 (2018) 013503.

[11] S. Zoletnik et al, Review of Scientific Instruments 89.6 (2018) 063503.

[12] M. Makansi, et al, J. Phys. Chem. 59, (1955) 40.

[13] R. Fischer et al, Plasma Physics and Controlled Fusion 50.8 (2008) 085009

[14] M. Vécsei et al, Edge density profile and turbulence 
measurements with an alkali beam diagnostic on Wendelstein 7-X, 45th EPS Conference on Plasma Phys.,

ECA Vol., P5.1002, 2018. 